\title{
Addresses of IMIA Member Societies
}

\section{American Medical Informatics Association (AMIA) \\ http://www.amia.org}

Office Address:

4720 Montgomery Lane, Suite 500

Bethesda, MD 20814 USA

Tel: + 13016571291

Fax: + 13016571296

E-mail:mail@amia.org

Twitter: @AMIAinformatics

Facebook: AMIA Informatix

\section{Argentine Association of Medical Informatics}

Asociación Argentina de Informática Médica (AAIM)

http://www.aaim.org.ar

\section{Office Address:}

Universidad Austral

Escuela de Medicina

Av. Pte. Peron 1500, ex ruta 234

Pilar - Buenos Aires Argentina 1664

Tel: + 542322482216

Fax: +54 2322482216

E-mail: info@aaim.com.ar

\section{Association for Health Informatics of Nigeria}

\section{Office Address:}

Medanchor Suite

264 Ikorodu Road Obanikoro Lagos, Nigeria 10007

Tel: + 23418972440

Fax: + 23414961378

E-mail: info@a-hin.org

\section{Association for Medical and Bio-Informatics, Singapore (AMBIS)}

http://www.ambis.org.sg

Office Address:

Eusoff Hall

10 Kent Ridge Drive

National University of Singapore

Singapore, Singapore 119242
IMIA Representative:

Karen Greenwood

4720 Montgomery Lane, Suite 500

Bethesda, MD 20814 USA

E-mail:karen@amia.org

Chair:

Dr Thomas Payne

University of Washington

Seattle, WA

USA
President and IMIA Representative

Alan David March, MD

Universidad del Salvador

Buenos Aires

Argentina

Tel: +5491147062321

E-mail: alandmarch@gmail.com
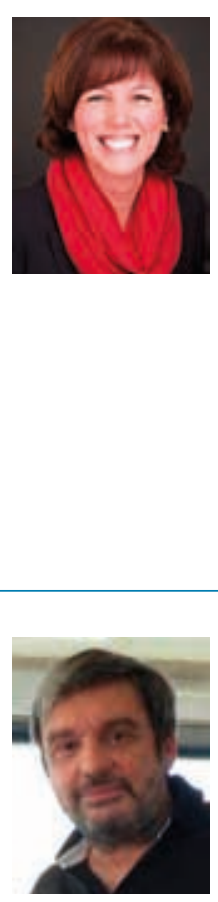

President and IMIA Representative:

Dr. Olusegun Odujebe

CEO Enthusia Consulting Ltd.

Lagos, Nigeria

Tel: +23418972440

E-mail: segunodujebe@gmail.com

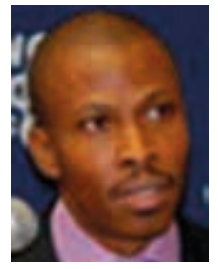

President and IMIA Representative:

Dr. Adam Chee

Chief Advocacy Office

BirneyHealthCare

VBox 889312

Singapore 919191

E-mail: query@binaryhealthcare.com

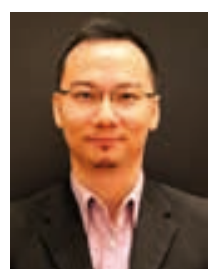




\section{Belgian Medical Informatics Association}

http://www.bmia.be

Office Address:

Rue Bon Air 2

1470 Basiy-Thy, Belgium B-4280

Tel: +3271287038

E-mail: p.piette@hopitaux-gilly.be
President:

Dr. Etienne De Clercq

Université Catholique de Louvain

1200 Brussels, Belgium

Tel: + 3227643262

E-mail: Etienne.Declercq@uclouvain.be

IMIA Representative:

Prof. Francis H. Roger France

Université Catholique de Louvain

Centre for Medical Informatics

10, av Hippocrate, Brussels, Belgium B-1200

Tel: +3223753748

E-mail: fhrf@rogerfrance.com
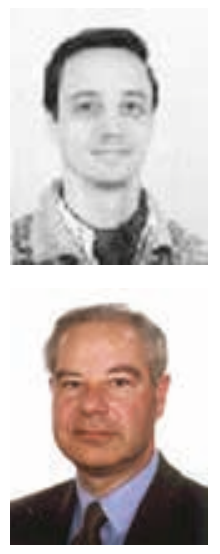

\section{Brazilian Society of Health Informatics}

Sociedade Brasileira de Informática em Saúde - SBIS

http://www.sbis.org.br

\section{SBIS Office Address:}

Rua Tenente Gomes Ribeiro, 57 - sala 33

Vila Clementino - SP

São Paulo, SP Brazil 04038-040

Tel: +551137913343

Fax: +551137913343

E-mail:sbis@sbis.org.br

\section{British Computer Society (BCS Health)}

http://www.bcs.org/category/6044

\section{Office Address:}

British Computer Society (BCS) Health Manager

1st Floor, Block D, North Star House North Star Avenue

Swindon, Wiltshire UK SN2 IFA

Tel: + 441793417635

E-mail: jonathan.jeffery@hq.bcs.org.uk
President and IMIA Representative:

Beatriz de Faria Leão

AV Macuco 550 apto 141

São Paulo, SP, Brazil

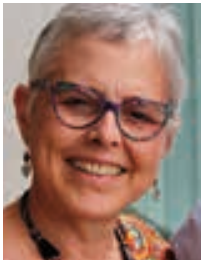

Chair:

Dr. Justin Whatling

Chair

BCS Health

Swindon,UK

Phone: +44 (0) 1793417635

\section{IMIA Representative:}

Dr. Paul Woolman

Interim Information Services Manager

NHS Forth Valley

Tel: + 44 (0) 1324678507

Mobile: +44 (0) 7584575497

E-mail: paul.woolman@nhs.net

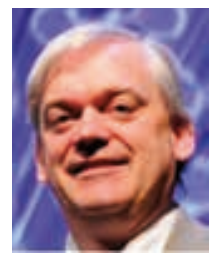




\section{Burundi Health Informatics Association}

Association Burundaise d'Informatique Medicale (ABIM)

Burundi Health Informatics Association (BHIA)

\section{Office Adress:}

ABIM

Avenue de I'ONU, 4

BP 1210, Bujumbura, Burundi

Tel: + 25722276772

Fax: + 25722254154

Email: info@abim-bhia.org

\section{Cameroonian Health Informatics Society (CAHIS)}

Société Camerounaise d'Informatique Médicale (SOCIM)

\section{Office Adress:}

Yaoundé Central Hospital

Faculty of Medicine and Biomedical Sciences

Yaoundé, Cameroon

\section{Chilean Health Informatics Society}

Asociación Chilena de Informática en Salud (ACHISA)

http://www.achisa.org

\section{Office Address:}

Napoleón 3565, of 202

Las Condes

Santiago, Chile

\author{
President: \\ Egide Njejimana \\ Tel: +25777746300 \\ E-mail:enjejimana@yahoo.fr \\ IMIA Representative
Dr. Frank Verbeke
Tel: + 25778470470
or +3247562 1569
E-mail:frank.verbeke@openit-burundi.net
}

President:

Prof. Vincent Diientcheu

Neurosurgeon

Yaoundé Central Hospital

Yaoundé, Cameroon

E-mail:vincent_diientcheu@yahoo.com

IMIA Representative

Mr. Ghislain B. Kovematchoua Tchuitcheu President \& CEO

Koegni-eHealth Innovation for Development

Hamburg, Germany

E-mail: ghislain.k@koegni-ehealth.org

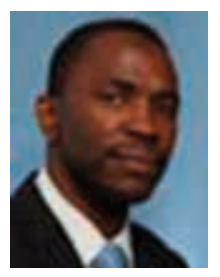

President:

Jaime de los Hoyos

Santigo, Chile

E-mail: jdeloshoyos@achisa.org

IMIA Representative:

Alejandro Mauro

E-mail: alemauro@gmail.com 


\section{China Medical Informatics Association (CMIA)}

China Medical Informatics Association of

Chinese Institute of Electronics

http://www.cmia.info

Office Address:

17 Zhengive Jiadao, Xinjiekou, Xicheng District

Beiiing 100035, China

Tel: + 861083221107

Fax:+86108322 1393

E-mail: abc@cmia.info

\section{Colombian Association of Health Informatics}

Asociación Colombiana de Informática en Salud (ACIESA)

\section{COACH, Canada's Health Informatics Association}

\section{http://www.coachorg.com}

Office Address:

250 Consumers Road, Suite 301

Toronto, Ontario M2J 2V6

Tel: +14164949324

Fax: + 14164958723

E-mail: info@coachorg.com

\section{Croatian Society for Medical Informatics (CSMI)}

Hrvatsko drustvo za medicinsku informatiku

http://www.hdmi.hr

\section{Office Address:}

Rockefellerova 4

Zagreb, Croatia

Tel: +3851 4590105

Fax: +38514684406

E-mail: jkern@snz.hr
Chair:

Mr Yongqin Huang

Chairman and Executive Vice President of CMIA; President

Chinese Institute of Electronics, Medical Informatics Branch

Beijing, China

E-mail: abc556677@163.net

IMIA Representative:

Dr. Yue (Emmet) Huang

Member of the Board of CMIA

Beijing, China

E-mail: hyuehyue@126.com

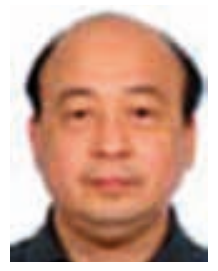

Chairman and IMIA Representative

Dr. Carlos Arteta Molina

Calle 163 a 13 b 60 Tower E 5th Floor

Bogota

Colombia

E-mail: carteta@gmail.com

President:

Mike Barron

President and Chief Executive Officer,

Newfoundland \& Labrador Centre for Health Information (NLCHI)

70 0'Leary Avenue, St. John's, NL A B 2 C7

Canada

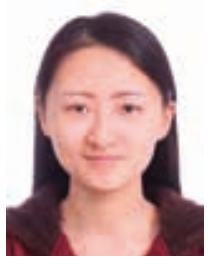

IMIA Representative:

Jeremy Theal, MD, FRCPC

Chief Medical Information Officer

North York General Hospital

4001 Leslie Street, Suite GS-70b

Toronto, Ontario, Canada M2K IEI

E-mail: jeremy.theal@nygh.on.ca

President:

Prof. Dr. Vesna llakovac

Assistant Professor

Josip Juraj Strossmayer University of Osijek

Osiiek, Croatia

Tel: + 38531512880

IMIA Representative:

Dr. Mira Hercigonja-Szekeres

Lecturer

Hrvatsko Zagorje Polytechnic

Krapina, Croatia

Tel: + 385 (0)1 6636500

E-mail: mira.hercigonja-szekeres@zg.t-com.hr
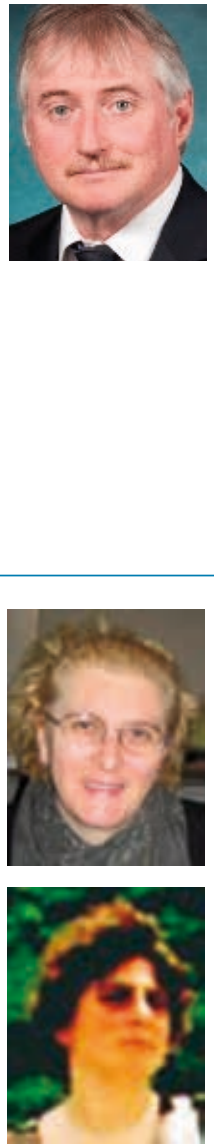


\section{Cuban Society of Medical Informatics}

Sociedad Cubana de Informática Médica

http://www.socim.sld.cu

Office Address:

CECAM

Calle 146 esq Ave 31 №. 2511 Cubanacán Playa

Ciudad de la Habana, Cuba

\section{Czech Society of Biomedical Engineering and Medical Informatics}

Ceska spolecnost biomedicinskeho inzenyrstvi a lekarske informatiky

http://www.cls.cz/

Office Address:

Czech Society,

Sokolska 33, Prague 2

The Czech Republic 12026

\section{eHealth Association of Pakistan (eHAP)}

Office Address:

Maria Arif

Coordinator eHAP

G-3A, Block 17 Gulshan-e-lqbal

Karachi, Pakistan

Tel: +92 2132009994

E-mail: Shaista.nazir@ehap.net.pk

\section{Finnish Social and Health Informatics Association (FinnSHIA)}

Sosiaali - ja terveydenhuollon tietojenkäsittely -yhdistys ry ry

http://www.stty.org
President and IMIA Representative:

Prof. Dr Esperanza O'Farrill

Medical Doctor

Havana University of Medical Science

Havana City

Cuba

Tel: +53721 1354

E-mail:espe@cecam.sld.cu

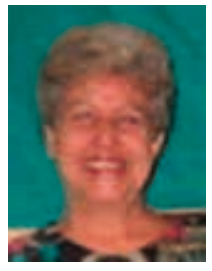

President:

Dr. Jiri Hozman

Head of Department

Czech Technical University in Prague

Kladno, Czech Republic

\section{IMIA Representative:}

Prof. Dr. Jana Zvárová

Professor \& Director, EuroMISE Center

Charles University and Academy of Sciences

Prague 8, Czech Republic

Tel: +420266053097

E-mail:zvarova@euromise.cz

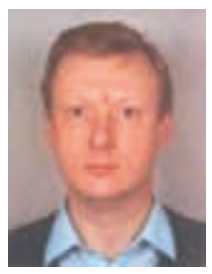

President:

Dr. Haroon Khan

Associate Professor and Consultant Pathologist

Pakistan Institute of Medical Sciences

Islamabad, Pakistan

IMIA Representative:

Dr. Hammad Durrani

General Secretary-eHAP

Manager Research, eHealth

Aga Khan University

Karachi, Pakistan

Tel: +92 2132009994

E-mail: hammad.durrani@aku.edu

Chair:

Mrs. Kristiina Häyrinen

Researcher

Kuopio, Finland

E-mail: kristiinahayrinen@gmail.com

IMIA Representative:

Dr Sari Palojoki

Chief Patient Safety Officer, PhD (health informatics)

Helsinki and Uusimaa University Hospital District

E-mail: sari.palojoki@hus.fi 


\section{French Medical Informatics Association (AIM)}

Association Française d'Informatique Médicale

http://france-aim.org

Office Address:

AlM Dr Pagonis

Secrétariat Pôle Santé Publique

Pavillon TAlLLEFER

CHU de Grenoble

CS1027 38043 Grenoble cedex 9, France

E-mail: contact@aim.org

\section{German Association for Medical Informatics, Biometry and Epidemiology}

Deutsche Gesellschaft für Medizinische Informatik, Biometrie und Epidemiologie (GMDS) e.V.

http://www.gmds.de

Office Address:

Industriestr. 154

Köln, Germany 50996

Tel: + 4922137994755

Fax: +4922137994756

E-mail: info@gmds.de

\section{Ghana Health Informatics Association}

Office Address:

Health Informatics Unit, Department of Biostatistics

College of Health Sciences, University of Ghana

P.O. Box LG 13, Legon

Accra, Ghana
President:

Jean Marie Rodrigues

E-mail: rodrigue@univ-st-etienne.fr

IMIA Representative:

Pr. Anita Burgun

Paris Descarte University

Paris, France

E-mail: anita.burgun@gmail.com
President:

Prof. Dr. sc. hum. Paul Schmücker

Hochschule Mannheim, Fakultät für Informatik

Institut für Medizinische Informatik

Paul-Wittsack-Str. 10

68163 Mannheim, Germany

E-mail: p.schmuecker@hs-mannheim.de

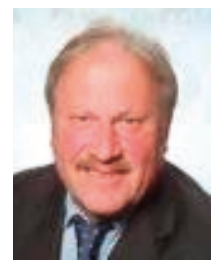

IMIA Representative:

Prof. Dr. Petra Knaup-Gregori

Universität Heidelberg

Institut für Medizinische Biometrie und Informatik

Sektion Medizinische Informatik

Im Nevenheimer Feld 305

69120 Heidelberg, Germany

Tel: +496221 56-7398 or + 49610185818

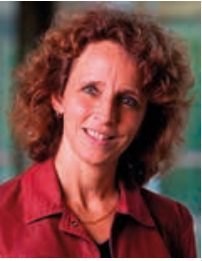

E-mail: Petra.Knaup@med.uni-heidelberg.de

President and IMIA Representative:

Dr. Wisdom Atiwoto

Department of Biostatistics, University of Ghana

P.O. Box LG 13, Legon

Accra, Ghana

Tel: + 233244333223

E-mail:wk.atiwoto@gmail.com

President and IMIA Representative:

Prof. John Mantas

Dean of the Faculty

University of Athens

Department of Nursing

11527 Athens, Greece

Tel: +30 210 7461459/60

E-mail: imantas@nurs.uoa.gr

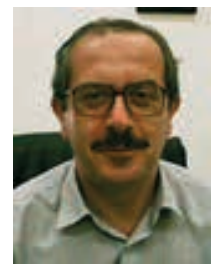

5, Navarchou Apostoli Street

Athens, Greece 


\section{Health Informatics New Zealand}

http://www.hinz.org.nz

\section{Office Address:}

P 0 Box 285

Albany Village

Auckland 0755

E-mail: admin@hinz.org.nz
Chair:

Lloyd McCann

E-mail: Lloyd@hinz.org.nz

IMIA Representative:

Dr. Inga Hunter

E-mail: i.hunter@massey.ac.nz

\section{Health Informatics Society of Australia Ltd. (HISA)}

http://www.hisa.org.au

Office Address:

1A/ 21 Vale Street

North Melbourne, Victoria Australia 3051

Tel: +6139326 3311, Fax: +61386100006

E-mail: hisa@hisa.org.au

\section{Health Informatics Society of Sri Lanka}

http://www.hissl.org

Office Address:

NOMA Office

$4^{\text {th }}$ Floor, Postgraduate Institute of Medicine

University of Colombo

Kynsey Road, Colombo 00800, Sri Lanka

Tel/Fax: + 94112689268

\section{Healthcare Informatics Society of Ireland}

\section{Cumann Riomheolais Sláinte}

http://www.hisi.ie

\section{Office Address:}

87-89 Pembroke Road

Dublin 4

Republic of Ireland

Tel: +3531 2377744

E-mail: info@hisi.ie
IMIA Representative and Chairperson:

Katerina Andronis

Director

Consulting in Life Sciences and Health Care, Deloitte

Melbourne, Australia

E-mail: hisa@hisa.org.au
President and IMIA Representative:

Prof. Vajira H. W. Dissanayake

Department of Anatomy, Faculty of Medicine

University of Colombo, 00800, Sri Lanka

Tel: +94 777351835

Fax: +942691 581

E-mail: vaijrahwd@hotmail.com

President:

Prof. Gerry Lyons

National University of Ireland, Galway

Galway, Ireland

E-mail: gerard.lyons@nuigalway.ie

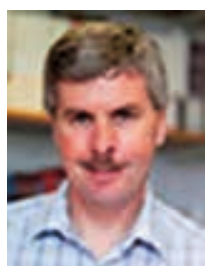

IMIA Representative:

Ms. Mary Sharp

Trinity College Dublin

Dublin, Ireland

Tel: +35318962732

E-mail: mary.sharp@scss.tcd.ie 


\section{Hong Kong Society of Medical Informatics}

http://www.hksmi.org

Office Address:

c/o Dr.Chun Por Wong

Ruttonjee Hospital

266 Quenn's Road East

Wanchai, Hong Kong, China

\section{Indian Association for Medical Informatics}

(IAMI)

http://www.iami.org.in

Office Address:

IAMI Head Office

c/o Mr Sushil Mehar

Dept of Computer Studies

AllMS Academic block, Ansari Nagar

New Delhi 110029, India

Tel: +91-11-26588332, 26594674 (PP for Mr Meher)

Mobile: +91-9868397023

\section{Iranian Medical Informatics Association}

http://www.IrMIA.org

Office Address:

P.O. Box 14665

Tehran, Iran 1411

\section{Ivorian Society of Biosciences and Health Informatics (ISBHI)}

\section{Societe Ivoirienne de Biosciences et d'Informatique} Medicale

\section{Office address:}

ISBHI

Focal Point for Réseau en Afrique Francophone pour la Télémédecine

RAFT-Côte d'lvoire

01 BP 2492, Abidjan, Côte d'Ivoire 01

Tel: + 22523537550

E-mail: fsehua@yahoo.fr
President and IMIA Representative:

Dr. Chun Por Wong

Chief of Integrated Medical Services

Ruttonjee Hospital

266 Queens's Road East

Wanchai, Hong Kong, China

Tel: + 85222911345

Fax: +852 2291 1335

E-mail: cpwong@ha.org.hk

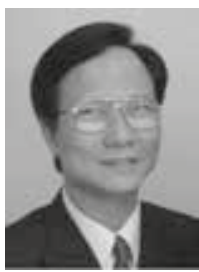

President:

Mr Sushil Mehar

Dept of Computer Facility

AllMS Academic block

Ansari Nagar

New Delhi 110029, India

E-mail:s meher@hotmail.com

IMIA Representative:

Dr. Shashi Bhushan Gogia

E-mail:gogia7@gmail.com
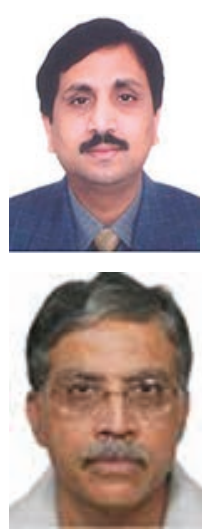

President and IMIA Representative:

Dr. Ramin Moghaddam

Director, Medical Informatics Department

Iranian Social Security Organization

Tehran, Iran

Tel: +982164502512

E-mail: Dr_Moghaddam@Medical-Informatics.Net

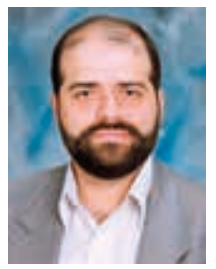

Chair:

Prof. Somian Francis Ehua

Head, Emergency Service

Yopougon University Hospital Center

Abibian, Côte d'lvoire

Tel: + 22523537550

E-mail: fsehua@yahoo.fr

IMIA Representative:

Dr. Innocent M. Nanan

Dental Surgeon

United Nations Operation for Côte d'Ivoire (UNOCI)

Abidjan, Côte d'lvoire

Tel: +22520236054

E-mail:shiny967@gmail.com 


\section{Japan Association for Medical Informatics}

http://jami.jp/english/

\section{Office Address:}

Boa Hongoh Building 8F 3-42-5

Hongoh Bunkyoku

Tokyo, Japan 113-0033

Tel: +81338121702

Fax: +81338121703

E-mail: office@jami.jp

\section{John von Neumann Computer Society}

Neumann János Számítógéptudományi Társaság

http://www.niszt.hu

Office Address:

Báthori u. 16.

Budapest, Hungary 1054

\section{Kenya Health Informatics Association}

http://www.kehia.org

\section{Office Address:}

Westlands, Waumini House, 2nd Floor

Nairobi, Kenya

E-mail: info@kehia.org

\section{Korea Society of Medical Informatics (KOSMI)}

http://www.kosmi.org
President:

Dr. Ryuichi Yamamoto

Graduate School of the University of Tokyo

Tokyo, Japan

Tel: +81358006550

E-mail:yamamoto@hcc.h.u-tokyo.ac.jp

IMIA Representative:

Prof Tomohiro Sawa

Teikyo University

E-mail: sawa@teikyo-masui.jp
President:

Prof. György Surián

IMIA Representative:

Prof. György Kozmann

Head, Dept. Information Systems

University of Pannonia

Budapest, Hungary

E-mail: kozmann@mfa.kfki.hu

President and IMIA Representative:

Mr Tom Oluoch

Tel: +254722 208924

E-mail: toluoch@ke.cdc.gov
President:

Prof. Jung Shin Lee

Professor, Department of Oncology

Asan Medical Center

University of Ulsan College of Medicine

Seoul, Korea

Tel: +82230103210

E-mail: jayslee@amc.seoul.kr

\section{IMIA Representative:}

Hyeoun-Ae Park

Professor, Biostatistics \& Health Informatics

College of Nursing

Seoul National University

Seoul, Korea

Tel: +8227408827

E-mail: hapark@snu.ac.kr

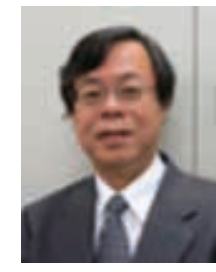




\section{Medical Informatics Association of Malawi (MIAM)}

Office address:

Box 149, Lilongwe, Malawi 3

E-mail: info@miam.org.mw

\section{Mexican Medical Informatics Association}

\section{Asociación Mexicana de Informática Médica} (AMIM)

http://www.amim.org.mx

\section{Office Address:}

Av. Conchita 3009

Col. Loma Bonita

Zapopan, Jal, Mexico 45086

\section{Norwegian Society for Medical Informatics}

\section{Norsk Helseinformatikk (NorHIT)}

http://www.fdh.no; www.helse.dnd.no

\section{Office Address:}

Den Norske Dataforening

PB 8874 Youngstorget

N-0028 0slo, Norway

E-mail: Janicke@dataforeningen.no

\section{Peruvian Association of Biomedical Informatics} http://www.apib.pe

\section{Philippine Medical Informatics Society, Inc.}

http://www.philmedinfo.org/

\section{Office Address:}

UP College of Medicine Medical Informatics Unit

547 Pedro Gil Street Ermita

Manila Philippines 1000

Tel: + 6325256501

Fax: +6325256501

E-mail: berdemd@yahoo.com
President and IMIA Representative:

Mr. Boster Sibande

Medical Informatics Association of Malawi

Lilongwe, Malawi

Tel: + 2658301959

E-mail: boster.sibande@yahoo.com

President:

Dr. Adrian Pacheco

Col Juarez, DF Mexico

Tel: +525552083939

E-mail:adrian.pacheco@salud.gob.mx

IMIA Representative:

Dr. Amado Espinosa

Guadalajara, Jal Mexico

Tel: +523330307030

E-mail: aespinosa@medisist.com.mx

\section{President:}

Dr. Anne Moen

Associate Professor, University of Oslo

Oslo, Norway

Tel: +4722840700

E-mail: anne.moen@intermedia.vio.no

IMIA Representative

Dr. Petter Hurlen

Chief, Department of Radiology

Akershus University Hospital

Lørenskog, Norway

E-mail: petter@hurlen.no
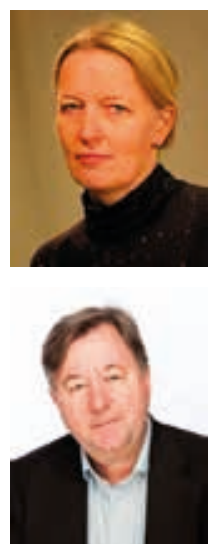

President and IMIA Representative:

Dr. Walter H. Curioso

E-mail:walter.curioso@upch.pe

President:

Dr. Erwin Brian Tan

Angeles City

Philippines

\section{IMIA Representative:}

Dr. Alvin Marcelo

Director UP Manila National Telehealth Center

Manila, Philippines

Tel: +6325256501

E-mail: alvin.marcelo@telehealth.ph

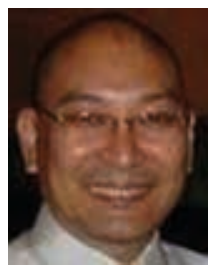




\section{Romanian Society of Medical Informatics}

\section{Societatea Romana de Informatica Medicala}

http://medinfo.umft.ro/rsmi/

Office Address:

Spl. T.Vladimirescu 14

Timisoara Timis, Romania 300173

Tel/Fax: + 40256490288

E-mail:mihalas@umft.ro

\section{Slovenian Medical Informatics Society (SIMIA)}

Slovensko drustvo za medicinsko informatiko (SDMI)

http://www.sdmi.si

\section{Office Address:}

Slovensko drustvo za medicinsko informatiko

c/o Katedra za javno zdravje

Zaloska 4, Ljubliana, Slovenia SI-1000

Tel: + 38615437540

Fax: +38615437541

E-mail: info@sdmi.si

\section{Society for Medical Informatics of Bosnia and Herzegovina}

http://www.bhsmi.ba

Office Address:

Faculty of Medicine

Department of Medical Informatics

University of Sarajevo

Sarajevo Cekalusa 90/IV

Bosnia and Herzegovina 71000

Tel: + 38733444714

Fax: +38733217271

\section{South African Health Informatics Association (SAHIA)}

http://www.sahia.org.za

\section{Office Address:}

2 Vinyard Avenue

Oakhurst Estate

Hout Bay, Cape Town

South Africa 7872
President and IMIA Representative:

Prof. Dr. George I. Mihalas

Professor

Victor Babes University of Medicine and Pharmacy

Timisoara, Romania

Tel: +40256490288

Fax: +40256490288

E-mail mihalas@umft.ro

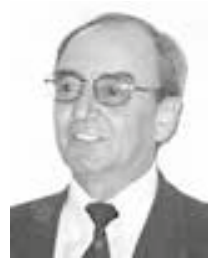

President:

Dr. Ivan Erzen

National Institute for Public Health

Ljubliana

Slovenia

E-mail: ivan.erzen@gmail.com

IMIA Representative:

Amdrej Orel

Marand d.0.0.

Jubliana

Slovenia

E-mail: Andrej.orel@marand.si
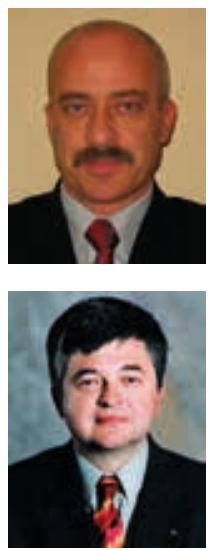

President and IMIA Representative:

Prof. Dr. Izet Masic

Medical Faculty

Sarajevo

Bosnia and Herzegovina

Tel/Fax: +38733444714

E-mail: imasic@lol.ba

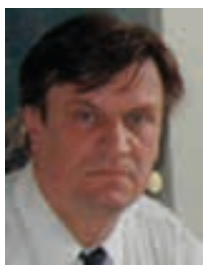

President:

Prof Nicky Mostert-Phipps

Nelson Mandela Metropolitan University

South Africa

E-Mail: nicky.mostert-phipps@nmmu.ac.za

IMIA Representative:

Dr. Lyn A. Hanmer

of Disease Research Unit and WHO Collaborating Centre

for the Family of International Classifications (WHO-FIC SA)

Medical Research Council

Tygerberg, Western Cape,South Africa

Tel: + 27219380343

Fax: +27219380315

E-mail:lyn.hanmer@mrc.ac.za

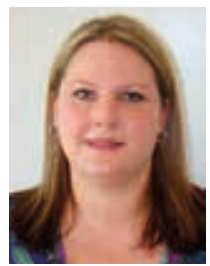




\section{Spanish Society of Health Informatics}

Sociedad Española de Informática de la Salud (SEIS)

http://www.seis.es

Office Address:

CEFIC (SEIS Secretary)

C/ Enrique Larreta

5. Bajo, Izda.

Madrid, Spain 28036
President:

Prof. Luciano Saez-Ayerra

Head of IT Dept.

Institute of Health Carlos III

Madrid, Spain

Tel: + 34913877835

E-mail: Isaez@isciii.es

IMIA Representative:

Carlos Luis Parra-Calderón

E-mail: carlos.parra.sspa@juntadeandalucia.es

President:

Professor emeritus Urban Forsum

IKE, Linköping universitet

58185 Linköping, Sweden

Tel: + 46705476128

E-mail: urban.forsum@liu.se

President and IMIA Representative:

Prof. Sabine Koch

Director, Health Informatics Centre

Karolinska Institutet

Stockholm, Sweden

Tel: + 46852487149

E-mail:sabine.koch@ki.se

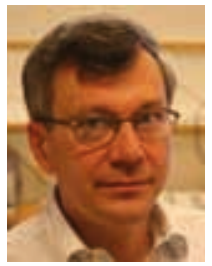

President:

Prof. Dr. Christian Lovis, MD, MPH

Head of the Division of Medical Information Sciences

University Hospitals of Geneva

CH-1211 Geneva 14, Switzerland

Tel: + 41223726201

E-mail: christian.lovis@hcuge.ch

IMIA Representative:

Prof. Dr. Antoine Geissbuhler

Geneva University Hospital

Chairman, Division of Heallth and Telemedicine

Geneva, Switzerland

Tel: + 4122372620

E-mail: antoine.geissbuhler@hcuge.ch
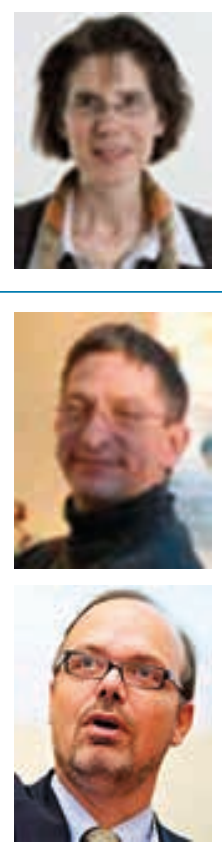

President:

Wang Prof. Da-Wei, PhD

Research Fellow, Institute of Information Science

Academia Sinica, Taiwan

120 Academia road, Settion 2 Nankang, Taipei 115, Taiwan

Tel: + 886-2-27883799\#1729

E-mail: wdw@iis.sinica.edu.tw

IMIA Representative:

Prof. Yu-Chuan (Jack) Li

Professor and Vice-President

Taipei Medical University

Taipei Taiwan

Tel: + 88622736 1661×2069

E-mail: jack@tmu.edu.tw

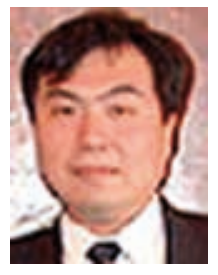




\section{Thai Medical Informatics Association}

http://www.tmi.or.th/

\section{Office Address:}

Bureau of Health Policy and Strategy

Office of Permanent Secretary

Ministry of Public Health

Thiwanon Road

Nonthaburi, Thailand 11000

\section{The Bolivian Medical Informatics and Telemedicine Society (SOBOTIM)}

SOciedad BOliviana de Telemedicina e Informática Médica

http://www.sobotim.org

Office Address:

Av. 15 de Abril \# 40, Villa Fátima

Le Paz - Bolivia

E-mail:info@sobotim.org

\section{The Israeli Association for Medical Informatics (ILAMI)}

Office Address:

P.0. Box 50043

Tel Aviv, Israel 61500

\section{The Mali Society of Biomedical and Health Information (SOMIBS)}

Société Malienne d'Informatique Biomédicale et de Santé (SOMIBS)

\section{hitp://www.keneya.net}

\section{Office Address:}

Hôpital Mère Enfant le Luxembourg

B.P. E3791

Hamdallaye, Bamako, Mali

E-mail:cob@keneya.net
President:

Dr. Choosna Makarasam

Department Consultant, Department of Medical Services

Ministry of Public Health

Nonthaburi, Thailand

Tel: + 6625918242

E-mail: choosna@gmail.com

IMIA Representative:

Dr. Siriwan Suebnukarn

Bureau of Policy and Strategy

Ministry of Public Health

Tiwanon Road, Nonthaburi, Thailand 11000

Tel: + 66 2590-1492

E-mail: siriwan.suebnukarn@gmail.com

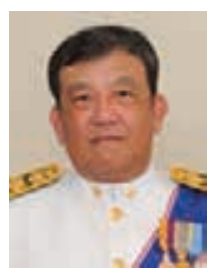

President:

Dr. Ramiro Narváez Fernández
President and IMIA Representative:

Dr. Batami Sadan

CEO

Vaica Medical Ltd

Tel-Aviv, Israel

Tel:+ 972777005388

E-mail: sadanba@netvision.net.il

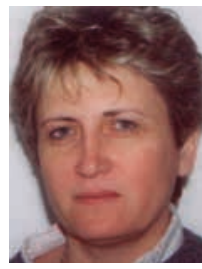

President:

Prof. Abdel Kader Traoré

Directeur CNAM

Université du Mali

Bamako, Mali

Tel: +2236746791

E-mail: aktraore@caramail.com

IMIA Representative:

Dr. Cheikh Oumar Bagayoko

Coordinator, RAFT

Université du Mali

Bamako, Mali

Tel: +2236750004

E-mail:cob281@yahoo.fr
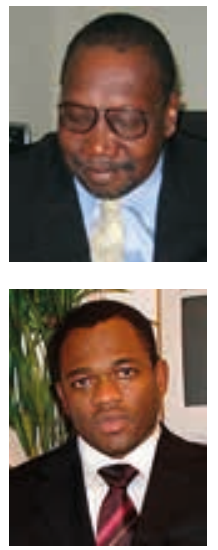


\section{The Saudi Association for Health Informatics (SAHI)}

http://www.sahi.org.sa

Office Address:

P.0.Box 90394

Riyadh 11613, Kingdom of Saudi Arabia

\section{The Ukrainian Association for Computer Medicine (UACM)}

http://www.uacm.kharkov.ua

\section{Office Address:}

P.0.Box 7313, 61002, Kharkiv, Ukraine

Tel: + 380577118032

Fax.: +380577118025

E-mail: Institute-MIT@ukr.net

\section{Togolese Association of Medical Informatics and Telemedicine (ATIM-TELEMED)}

Office Address:

BP1585

Lome, Togo

\section{Turkish Medical Informatics Association (TURKMIA)}

\section{Tip Bilisimi Dernegi}

http://www.turkmia.org

\section{Office Address:}

Bayraktar Mah. Bayrakli Sok. №: 39/2

Buyukesat

Ankara, Turkey

Tel: +903124 463531

Fax: +903124463586

E-mail: turkmia@turkmia.org
President and IMIA Representative:

Dr. Bandar S. Al-Haqbani

Tel: +9661 $2520088 \times 43447$

E-mail:alhaqbaniba@ngha.med.sa

\section{IMIA Representative:}

Prof. Oleg Yu. Mayorov

Chief Medical Informatician of Ukraine

Ministry of Healthcare

Kharkiv Medical Academy of Postgraduate Education

Kharkov, Ukraine

Tel: +380 577118032

E-mail: snd@ic.kharkov.va

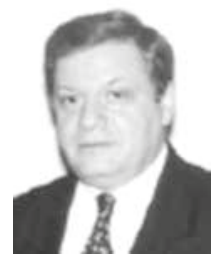

President and IMIA Representative:

Mr. Koumaada Walimba Badombena-Wanta

President

ATIM-TELEMED

Lome, Togo

Tel: +2289113811

E-mail: atim.telemed@gmail.com

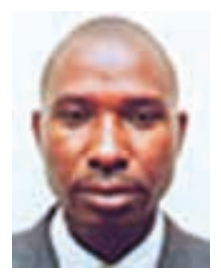

President:

Dr. Mustafa Ozmen

Professor of Radiology

Hacettepe University School of Medicine

Ankara, Turkey

Tel: +903123051 101

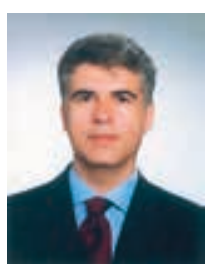

\section{IMIA Representative:}

Dr. K. Hakan Gülkesen

Assistant Professor

Akdeniz University, Antalya

Tel: +90532775 7910

E-mail: hgulkesen@akdeniz.edu.tr

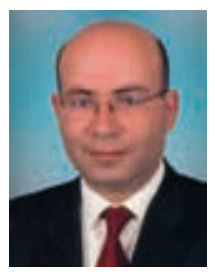




\section{Uruguayan Society of Health Informatics}

Sociedad Uruguaya de Informática en la Salud (SUIS)

http://www.suis.org.uy

Office Address:

Bvar. Artigas 1515

Montevideo Uruguay CP 11600

\section{Venezuelan Association of Computer Science in Health (AVIS)}

http://www.avis.org.ve

Office Address:

Urb Zapara I, Block 11 B behind the Church

Maracaibo, Zulia, Venezuela

Tel: +584247268866

E-mail:avis.org@gmail.com

\section{VMBI, Society for Healthcare Informatics, The Netherlands}

Vereniging voor informatieverwerking in de zorg (VMBI)

http://www.vmbi.nl

Office Address:

VMBI

Utrechtseweg $129 \mathrm{~A}$

3702 AC Zeist

The Netherlands

E-mail: info@VMBI.nl
President:

Dr. Julio Leivas

British Hospital

Montevideo, Uruguay

Tel: +59895262192

E-mail: |leivas@adinet.com.uy

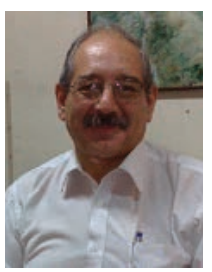

IMIA Representative:

Dr. Jorge Stanham

Medical Advisor

British Hospital

Montevideo

Tel: +59824871020

E-mail: jstanham@mednet.org.uy

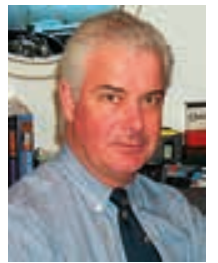

President and IMIA Representative:

Prof. Dr. Osman Arguello

President, Technology, Health and Creativity CA

Coordinator of the Regional Information System Health from

Tachira State

Maracaibo, Estado Zulia, Venezuela

Tel: +584125349531

E-mail: osmanarguello@gmail.com

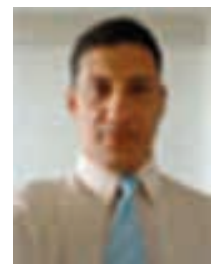

Chair:

Mr. Jo Bollen, MSc

E-mail: jo.bollen@vmbi.nl

IMIA Representative:

Dr. Ronald Cornet

Academic Medical Center, University of Amsterdam

Amsterdam, The Netherlands

Tel: +31205665188

E-mail: R.Cornet@amc.uva.nl 


\section{Working Group Medical Informatics and eHealth of the Austrian Computer Society (OCG) and the Austrian Society for Biomedical Engineering (ÖGBMT)}

Arbeitskreis Medizinische Informatik (AKMI) der Österreichischen Gesellschaft für Biomedizinische Technik (ÖGBMT) und der Österreichischen Computergesellschaft (ÖCG)

http://iig.umit.at/akmi/akmi.htm

Office Address:

Reininghausstr. 13/1

A-8020 Graz, Austria

Tel: +4331658657011

Fax: + 4331658657012

E-mail: guenter.schreier@ait.ac.at
President:

DI Dr. (habil) Günter Schreier

AlT Austrian Institut for Technology

Reininghausstr. 13/1

8020 Graz, Austria

Tel: 4331658657022

E-mail: guenter.schreier@ait.ac.at

\section{IMIA Representative:}

Prof. Dr. Elske Ammenwerth

Professor, Institute for Health Information Systems

University for Health Sciences, Medical Informatics and

Technology

Tirol, Austria

Tel: + 435086483809

E-mail: Elske.Ammenwerth@umit.at
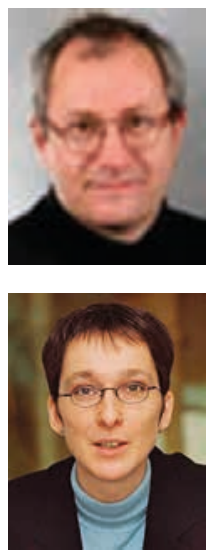